Table 2 Mean (SD) CSF aminoacid concentrations in five patients with $A L S$ at diagnosis and one year later ( $\mu \mathrm{mol} / \mathrm{l})$

\begin{tabular}{lcc}
\hline & $\begin{array}{l}\text { Time of } \\
\text { diagnosis }\end{array}$ & $\begin{array}{l}\text { One year } \\
\text { later }\end{array}$ \\
\hline Aspartic acid & 0 -trace & 0 -trace \\
Asparagine & $5 \cdot 0(1 \cdot 3)$ & $9 \cdot 6(1 \cdot 1)^{\star \star}$ \\
Glutamic acid & $0 \cdot 8(0 \cdot 3)$ & $0 \cdot 8(0 \cdot 3)$ \\
Serine & $10 \cdot 7(3 \cdot 6)$ & $14 \cdot 5(2 \cdot 1)$ \\
Glutamine & $289(29)$ & $257(30)$ \\
Taurine & $3 \cdot 4(0 \cdot 8)$ & $4 \cdot 2(0 \cdot 8)$ \\
Alanine & $19.9(2 \cdot 1)$ & $23.5(4 \cdot 6)$ \\
Tryptophan & $0.4(0 \cdot 2)$ & $1.3(0 \cdot 3)^{\star \star}$ \\
Valine & $9 \cdot 6(3 \cdot 6)$ & $23.5(4 \cdot 6)^{\star \star}$ \\
Phenylalanine & $8.5(2 \cdot 4)$ & $17 \cdot 4(3 \cdot 1)^{\star \star}$ \\
Isoleucine & $3.9(1 \cdot 2)$ & $7 \cdot 3(1 \cdot 4)^{\star}$ \\
Leucine & $10.5(3 \cdot 7)$ & $17.9(3 \cdot 8)^{\star}$ \\
\hline
\end{tabular}

${ }^{\star} \mathrm{p}<0.05,{ }^{\star \star} \mathrm{p}<0.01$ according to the Mann Whitney U test.

in trace amounts in the CSF of the patients.

One year after the diagnosis of the disease, an increase was observed in the asparagine, isoleucine, leucine, phenylalanine and tryptophan CSF concentrations (table 2). The mean valine concentration decreased. Aspartic acid and glutamic acid levels were not affected. These results fit previous data showing that an increase occurred in the phenylalanine, isoleucine, leucine, valine, isoleucine, leucine, and tryptophan CSF levels of ALS patients. ${ }^{3}$ Unlike these authors, however, we did not observe any significant differences in the lysine, alanine, ${ }^{34}$ arginine, glutamine ${ }^{3}$ or serine ${ }^{4}$ levels.

These results might be mainly attributable to a general deterioration in the patients' condition, decreased activity, or abnormalities in their nutritional status. A general deterioration of the metabolic status may also have been responsible. Although none of our patients had any biological marks of hepatic failure, ultrastructural abnormalities of ALS patients' hepatocytes have been reported ${ }^{5}$ and hepatic failure may be correlated with an increase in brain phenylalanine, tryosine and tryptophan.

Several factors have been suggested to account for the discrepancies between the published data: the total glutamate and asparate concentrations measured in the control subjects differ tremendously between Perry's ${ }^{3}$ and Rothstein's ${ }^{4}$ studies. Using the HPLC technique our results with the neurological controls were of the same order of magnitude as those reported on healthy volunteers by other authors.

We did not observe any significant changes in the CSF glutamate or asparate levels in our patients who had been symptomatic for 13 months at the time of diagnosis and for 25 months at the time of the second CSF analysis. Furthermore, like Perry, ${ }^{3}$ we did not observe any significant correlations between the CSF glutamate levels and age. This may suggest that the neuroexcitatory toxic mechanism, if it occurs, mostly takes place early in the course of the disease, probably before the onset of clinical ALS, and that the discrepancies between studies on the CSF glutamate levels may not be attributable only to the progression of the disease in the patients tested.

At the end-stage of the disease, the anterior horn motor neurons degenerate and the spinal glutamate concentration decreases. ${ }^{2}$ This decrease is usually correlated with the loss of glutamatergic nerve terminals. The loss of glutamatergic afferents might be due to an excess of glutamate, and glutamatergic neurons might become irreversibly damaged before the onset of clinical ALS. Due to the decreased tissue glutamate levels, one might have expected to observe a decrease in the CSF glutamate level. Since the glutamate CSF level remained normal ${ }^{3}$ or became elevated in some patients that had been symptomatic for 15 months, ${ }^{4}$ glutamate turnover may increase in the surviving neurons.

We thank Professor J Pouget and Professor C Desnuelle who kindly referred several patients with ALS. We are grateful to the patients with ALS who provided CSF specimens. The English revision was carried out by Dr J Blanc. Médicale et Clinique, CHU Timone, 13385 Marseille Cedex 5, France D SAMUEL

Cellular and Functional Neurobiology Unit, CNRS, 31 Ch foseph Aiguier, 13009 Marseille, France G SERRATICE

Clinique des Maladies du Système Nerveux et de l'Appareil Locomoteur, CHU Timone, 13385 Marseille Cedex 5, France

Correspondence to: $\mathrm{Dr}$ Blin, Laboratoire de Pharmacologie Médicale et Clinique, CHU Timone, 13385 Marseille Cedex 5, France.

1 Plaitakis A, Caroscio JT. Abnormal glutamate metabolism in amyotrophic lateral sclerosis. Ann Neurol 1987;22:575-9.

2 Plaitakis A, Constantakakis E, Smith J. The neuroexcitotoxic amino acids glutamate and aspartate are altered in the spinal cord and brain in amyotrophic lateral sclerosis. Ann Neurol 1988;24:446-9.

3 Perry TL, Krieger C, Hansen S, Eisen A Amyotrophic lateral sclerosis: amino acid levels in plasma and

4 Rothstein JD, Tsai G, Kunc R, et al. Abnormal excitatory amino acid metabolism in amyotrophic lateral sclerosis. Ann Neurol 1990;28:18-25.

5 Nakano Y, Hirayama K, Terao K. Hepatic ultrastructural changes and liver dysfunction in amyotrophic lateral sclerosis. Arch Neurol 1987;44:103-6.

Selective enhancement of executive function by idazoxan in a patient with dementia of the frontal lobe type

The coeruleocortical noradrenergic system has long been implicated in the process of focused attention and more recently in controlled or effortful processes, and the allocation of attention. ${ }^{1}$ Broadly speaking the prefrontal cortex seems to be essential for tasks that require directed attention and effortful processing - tasks such as reasoning, anticipation, goal establishment, planning, organisation of behaviour, and monitoring of feedback. These terms are often subsumed under the heading "executive function". Psychopharmacological studies in experimental primates and humans have implicated the noradrenergic system as an important modulator of frontal lobe function..$^{234}$ These behavioural observations are supported by neuroanatomical ones, as there is a high density of noradrenergic $\alpha_{2}$ receptors in the frontal cortex. Two recent reports have suggested that it may be possible to improve performance on tests of frontal function using noradrenergic drugs such as the mixed $a_{1} / a_{2}$ adrenoceptor agonist clonidine, and the more selective $a_{2}$ adrenoceptor antagonist idazoxan. ${ }^{34}$

We report enhancement of executive function by idazoxan in a male patient with mild dementia of the frontal lobe type. A consultant neurologist (JRH) made a diagnosis of frontal type dementia, supported by the result of his SPECT scan, which showed right frontal hypometabolism. On neuropsychological assessment, he was found to have a relatively specific pattern of frontal dysfunction. For example, his score on the mini mental state examination was normal $(29 / 30)$ whereas his score on verbal fluency was severely impaired compared with that of normal volunteers, and comparable with that of neurosurgical patients with frontal lobe excisions. A $40 \mathrm{mg}$ oral dose of idazoxan was given to the patient on two occasions with a double-blind, placebocontrolled protocol. Test sessions were separated by at least a $\mathbf{4 0}$ hour washout period, and an ABBA design was used so as to counterbalance any order effect. A range of computerised tests measuring attention, memory, and planning was given, in an attempt to pinpoint a selective effect of idazoxan on tests with a large executive component, implicating the frontal lobes. This dose was well tolerated and except for transient nausea and a slight increase in blood pressure $(25 / 15 \mathrm{mmHg})$, no adverse reactions occurred.

A selective improvement was found. Specifically, idazoxan improved the efficiency of planning by reducing the number of excess moves made on the Tower of London test of planning (figure). Furthermore, idazoxan increased the patient's verbal fluency for categories and also the percentage of correct detections made on a rapid visual information processing (RVIP) test of sustained attention. The Tower of London and verbal fluency have been shown to be sensitive to frontal lobe dysfunction, and current investigations in our own laboratory suggest RVIP is another such test. By contrast, no change in performance was found for tests of paired associates learning, pattern and spatial recognition, or digit span. Such a selective pattern of cognitive enhancement supports

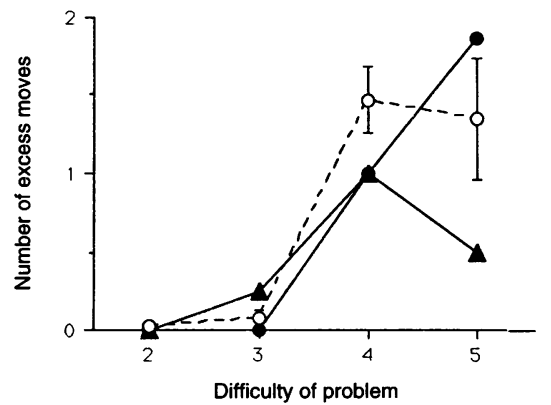

Figure Beneficial effect of idazoxan ( $\mathbf{\Delta}$ ) compared with placebo (O) on problem-solving performance in the Tower of London test of planning, in a patient with dementia of the frontal lobe type. Performance is measured as the number of excess moves required to solve the problem; the lower the excess moves, the better the performance. Difficulty of problem refers to whether a problem requires a minimum of $2,3,4$, or 5 moves for completion. The control data $(\mathrm{O})$ were obtained from a sample of volunteers $(n=12)$ matched to the patient for age, sex and estimated premorbid $I Q$. Bar $=S E$ 
the hypothesis that $\alpha_{2}$ agents affect frontal lobe functioning, and provides a rationale for the pharmacological treatment of frontal dementias. This preliminary report in a single patient awaits extension to a larger sample.

This research was supported by a Wellcome Trust Programme grant to Dr TW Robbins, BJ Everitt, and BJ Sahakian. We thank Ms GM Charlesworth for assistance with testing, Dr TW Robbins for critical reading of the manuscript, and Pierre Fabre Medicament for the idazokan.

B J SAHAKIAN J J COULL Departments of Experimental Psychology and Neurology, University of Cambridge, Downing Site, Cambridge CB2 $3 E B$, UK

Correspondence to: Dr Hodges

1 Cole BJ, Robbins TW. Forebrain norepinephrine: role in controlled information processing in the rat. Neuropsychopharmacology 1992;7:129-42.

2 Arnsten AFT, Contant TA. Alpha-2-adrenergic agonists decrease distractability in aged monkeys performing the delayed response task. Psych

task. Psychopharmacology 1991;108:159-69. offoot A, O'Carroll R, Ebmeir KP, Goodwin GM. Effects of clonidine on cognition and CBF in Korsakoff's psychosis BAP meeting, York, 21-24 July, 1991, abstract 24

4 Smith AP, Wilson SJ, Glue P, Nutt, DJ. The effects and after-effects of the alpha-2adrenoceptor antagonist idazoxan on mood, memory and attention in normal volunteers. f Psychopharmacol 1991;6:376-82.

5 Goldman-Rakic PS, Lidow MS, Gallager DW. Overlap of dopaminergic, adrenergic and serotonergic receptors and complementarity of their subtypes in primate prefrontal cortex. F Neurosci 1990;10:2125-38

\section{Ictal language shift in a polyglot}

There are a number of dysfunctions of language associated with epilepsy. During an episode usually associated with a temporal lobe focus patients may have loss of speech, ${ }^{1}$ dysphasia, ${ }^{2}$ or automatisms. ${ }^{3}$ A patient is described who speaks six languages and has automatisms in a number of these languages. She is a 49 -year-old Indian woman with no history of major illness, loss of consciousness, or head trauma. The episodes first began in 1980. She was speaking English and suddenly spoke a few words of Gujurati that were totally out of context. She did not remember what she said and had no change of consciousness associated with this. The patient was unwell at the time with a urinary tract infection. The second episode was in 1991, when she had an episode during which she shifted from Punjabi to English but did not remember the context. There have been fupur further episodes in the past year. On one occasion she was on a telephone speaking to a sister in Punjabi and said in Gujurati "I don't know, there is nothing we have cooked." She does not remember anything about this conversation but remembered what she had said previously and carried on with further conversation normally. On another occasion she again spoke in Gujurati “we don't have clothes". On a further occasion she switched from Gujurati to Urdu, saying "the closet is empty, there is nothing in the closet." The conversation ras continued for a short time by the sister out the patient could not remember this. The last episode was while teaching in English, she spoke Punjabi.

Some of these episodes have been

observed by her mother who is fluent in all these languages and noticed that the patient had flickering eyes and gulping.

The patient speaks Punjabi at home and speaks English and Swahili commonly. She also learnt Gujurati as a child, is able to read and write Hindi, and speaks Urdu.

On examination the patient was alert and orientated. There were no abnormalities on neurological examination. An EEG showed bilateral temporal lobe spikes, more on the right, which were accentuated with overbreathing. A CT scan was normal. The patient was given $100 \mathrm{mg}$ carbamazepine twice a day and no further episodes occurred during the ensuing four months.

A variety of speech disturbances may occur in patients with temporal lobe epilepsy. Dysphasia is associated with seizures originating in the dominant hemisphere..$^{3}$ Investigations to localise the disturbances have been made by stimulation studies. ${ }^{34}$ Speech automatisms have usually been localised to the temporal lobe of the nondominant hemisphere, although they are occasionally noted in the dominant hemispheres. ${ }^{1}$

Much information exists on the speech disturbances in multilingual people with strokes. There are two main themes, one propounded by Ribot, who said that the first language to be acquired is the first to recover and one by Pitres, who proposed that the language most familiar and most often used, will return first. ${ }^{2}$ The emotional attachment to a language is also important. ${ }^{2}$ Recovery may be parallel, when all the languages recover at a similar rate, which is the most common, differential, or successive. The patient may even develop a foreign accent after a cerebral infarction. In a study of bilingual patients given stimulation there can be a dissociation of sites where naming is affected. ${ }^{4}$ There are only rare reports of language shift in epileptic patients. In a patient undergoing stimulation of the right temporal lobe, ${ }^{3}$ the patient said "je suis malade" later followed by "I seem to lose my memory". Another patient, who was a Polish and English speaker, had as a seizure phenomenon the utterance "I beg your pardon" which occurred during a Polish conversation; this was also provoked by metrazol. ${ }^{1}$

The patient described here had speech automatism in a variety of Asian languages and English. Cases have been reported of language problems in Chinese-English speakers with strokes but reports for Indian-English speakers are rare. One patient spoke Telugu, Kannada, Tamil, and English fluently, but after a stroke had pure alexia in Kannada and English. ${ }^{5}$

The mechanism for shift of languages is not clearly understood. It has been postulated that it is due to a lesion of the supramarginal gyrus, but this holds true in only rare cases. The switching of languages is probably more of a generalised than specific neural mechanism. ${ }^{2}$ Automatisms, as in our patient, are more localised to the temporal lobes and usually the non-dominant hemisphere.

\section{MARTIN S SCHWARTZ Department of Neurology, Department of Neurology,
Atkinson Morley's Hospital, Copse Hill, Winbledon SW20 ONE, UK}

1 Serafetinides EA, Falconer MA. Speech disturbances in temporal lobe seizures; a study in 100 epileptic patients submitted to anterior temporal lobectomy. Brain 1963;
2 Paradis M, Lecours PR. Aphasia in bilinguals and polyglots. In: AR Lecours, F Lhermitte, B Bryans, eds. Aphasiology. London Bailliere Tindall, 1983:455-64.

3 Feindel W, Penfield W. Localization of discharges in temporal lobe automatisms. AMA Arch Neurol 1954;72:605-30.

4 Ojemann GA. Brain organisation for language from perspective of electrical stimulation mapping. Behav Brain Sci 1983;6:1 189-230.

5 Karanth P. Pure alexia in a Kannada-English bilingual. Cortex 1981;17:187-98.

Relief of trigeminal neuralgia by proparacaine

Two recent publications ${ }^{12}$ have reported the successful treatment of trigeminal neuralgia by the ophthalmic anaesthetic proparacaine hydrochloride $0.5 \%$ instilled in the eye of the affected side.

We tried this medication in 15 patients suffering from neuralgia involving one or two branches of the trigeminal nerve. In three patients there was involvement of the second division of the nerve, whereas in eight, the first and second divisions were affected. The other four patients complained of pain in the distribution of the second and third divisions of the nerve. In 13 patients the neuralgia was idiopathic; all had been taking carbamazepine for a considerable time with partial or no response. One patient had a giant suprasellar aneurysm and another a large acoustic neuroma, both inoperable because of the patients' advanced age and general condition.

The treatment consisted of instillation of two drops of proparacaine hydrochloride $0.5 \%$ in the eye of the affected side, in every case. A satisfactory effect was obtained in 13 patients with a clear cut improvement of symptoms allowing withdrawal or reduction of the daily dose of carbamazepine. In two patients (including a hospital physician) the medication stopped an attack of severe pain and this result is now permanent. Instillation was repeated once in eight patients and twice in two patients to obtain a stable and lasting result. The observation period ranged between one and four months and no side effects were reported by the patients. One patient experienced no change in her symptoms after the initial instillation and refused a second attempt, and another patient was lost to follow up.

In accordance with the previous authors, we find it difficult to propose a mechanism by which a benzoic ester with topical anaesthetic effect produces lasting relief of neuralgia in the distribution of the divisions of the trigeminal nerve that differ from that on which the drug acts directly. Is it possible that some of the drug drained through the lacrimal duct in the nasal and oral cavities, was absorbed by the mucosa and the nerve endings of the second division of the nerve, and eventually reached the trigeminal nucleus via retrograde axonal transport?

J VASSILOUTHIS Neurosurgical Department,

'Hygeia' Hospital, Kifissias Ave and Erythrou Stavrou Street, 15133 Athen

Greece

1 Zavon M, Fichte CM: Trigeminal neuralgia relieved by ophthalmic anaesthetic. $\mathcal{F} A m$ Med Assoc 1991;265:2807.

2 Spaziante R, Cappabianca P, Saini $M$, et al. Treatment of trigeminal neuralgia by ophthalmic anaesthetic. $\mathcal{F}$ Neurosurg 1992;77. 159-60. 